\title{
Preemptive analgesic efficacy of ultrasound-guided transversus abdominis plane block in patients undergoing gynecologic surgery via a transverse lower abdominal skin incision
}

\author{
Hyun-Jung Shin ${ }^{2}$, Sang Tae Kim ${ }^{1}$, Kyoung Hoon Yim ${ }^{1}$, Hyun Su Lee ${ }^{1}$, Jae Hwan Sim ${ }^{1}$, and \\ Young Duck Shin ${ }^{1}$
}

Department of Anesthesiology and Pain Medicine, ${ }^{1}$ College of Medicine, Chungbuk National University, Cheongju, ${ }^{2}$ Seoul National University Bundang Hospital, Seongnam, Korea

Background: The transversus abdominis plane block is recently described peripheral block to providing analgesia to the anterior abdominal wall. The goal of this study is to evaluate the analgesic efficacy of the ultrasound-guided transversus abdominis plane block (US-TAP block) in patients undergoing gynecologic surgery via a transverse lower abdominal skin incision.

Methods: Thirty-two patients undergoing gynecologic surgery were randomized to undergo standard care such as PCA, or to receive additional US-TAP block with standard care. After general anesthesia induction, a bilateral USTAP block was performed using $0.375 \%$ ropivacaine $20 \mathrm{ml}$ on each side. Postoperative demand of rescue analgesics in PACU and ward were recorded. Each patient was assessed postoperatively by a blinded investigator in the postanesthesia care unit (PACU) and at 2, 6, 10, 24, $48 \mathrm{hr}$ postoperatively to investigate pain, drowsiness, nausea and itch.

Results: The US-TAP block reduced pain intensity compared to standard care in the PACU (5.2 \pm 3.1 vs $8.4 \pm 1.3$ ) and at 2,24 postoperative hours ( $3.0 \pm 2.4$ vs $5.2 \pm 2.4,0.9 \pm 1.5$ vs $2.2 \pm 1.9$ ). Fentanyl requirements in PACU was reduced $(20.3 \pm 20.9$ vs $62.5 \pm 35.4 \mu \mathrm{g}, \mathrm{P}<0.05)$. In ward, pethidine requirements was reduced $(21.9 \pm 28.7 \mathrm{vs} 56.3 \pm 34.8 \mathrm{mg}$, $\mathrm{P}<0.05)$.

Conclusions: The US-TAP block with standard care provide more effective analgesia after gynecologic surgery via a transverse lower abdominal skin incision. (Korean J Anesthesiol 2011; 61: 413-418)

Key Words: Anesthesia, Nerve block, Ultrasonograpy.

Received: December 10, 2010. Revised: 1st, February 1, 2011; 2nd, March 9, 2011; 3rd, April 29, 2011. Accepted: May $29,2011$.

Corresponding author: Young Duck Shin, M.D., Department of Anesthesiology and Pain Medicine, College of Medicine, Chungbuk National University, 410, Sungbong-ro, Heungdeok-gu, Cheongju 361-763, Korea. Tel: 82-43-269-6234, Fax: 82-43-272-0264, E-mail: yydshin@cbnuh.or.kr (c) This is an open-access article distributed under the terms of the Creative Commons Attribution Non-Commercial License (http:// creativecommons.org/licenses/by-nc/3.0/), which permits unrestricted non-commercial use, distribution, and reproduction in any medium, provided the original work is properly cited. 


\section{Introduction}

The great majority of patients scheduled to undergo surgery suffer from emotional stress due to anxiety about the pain which is expected in the postoperative period [1]. Moreover, pain which is not treated promptly after surgery can impair the patient's ability to ambulate which may lead to adverse effects such as thromboembolism, myocardial ischemia, arrhythmia $[2,3]$.

Unceasing efforts have been tried to control the postoperative pain effectively. Multimodal techniques consist of a combination of opioids (either systemic or neuraxial), nonsteroidal anti-inflammatroy drugs and acetaminophen as well as patient-controlled analgesia (PCA) are currently used for pain control after surgery. But there are concerns with regard for the potential of systemically administered opioids to cause nausea, vomiting, drowsiness and respiratory depression [4]. Further, epidural patient-controlled analgesia which delivers the analgesics through a catheter placed into the epidural space can develop serious complications such as epidural abscess, meningitis, vertebral canal hematoma, spinal cord ischemia and paraplegia $[5,6]$.

Recently, a peripheral nerve block has been implemented to alleviate the problems above in addition to controlling the postoperative pain effectively at the same time and successful cases have been reported [7,8]. Especially, the use of ultrasound-guided nerve block has increased and ultrasound guidance offers the advantage of direct visualization of the needle and the anatomical structures. Therefore, one can see real time images during the procedure and for that reason, drugs can be injected more accurately and rapidly into the target site than the use a nerve stimulator or blindly blocking peripheral nerves. Ultimately, this enhances the safety and efficacy of the procedure [9].

A transversus abdominis plane (TAP) block is the technique to block the sensory nerves of the anterior abdominal wall and the TAP block has been used to control the pain after abdominal surgery in many cases [10-15]. It may replace the abdominal field block method which involves injection of local anesthetics into the lumbar triangle of Petit was introduced by Rafi [16] for the first time.

In this study, we evaluated the degree of pain and additional analgesic requirements when the ultrasound-guided transversus abdominis plane block (US-TAP block), as part of a multimodal analgesic regimen, was performed in patients undergoing gynecological surgery through a transverse lower abdominal skin incision. This was compared to standard care including patient-controlled analgesia with nonsteroidal antiinflammatory drugs and opioids.

\section{Materials and Methods}

This study was performed after obtaining approval from the Hospital Ethics Committee, and written informed consent from the patients with an explanation regarding the purpose, methods, effects, and complications. We studied 32 ASA physical status I, II patients scheduled for gynecological surgery under general anesthesia using a transverse lower abdominal skin incision (Cherney or Pfannenstiel incision). Patients were excluded if there was a history of relevant local anesthetics allergy, were currently using analgesics regularly for underlying disease such as arthritis, or if a body mass index (BMI) was higher than $35 \mathrm{~kg} / \mathrm{m}^{2}$.

Patients were randomly allocated into two groups: one group to undergo general anesthesia but not perform the US-TAP block (Group Control, $\mathrm{n}=16$ ) and the other group to undergo $0.375 \%$ ropivacaine injection, $20 \mathrm{ml}$ on the left and right side for a total of $40 \mathrm{ml}$ (Group US-TAP Block, $\mathrm{n}=16$ ).

None of the patients was premedicated before entering the operation room. On arrival in the operating room, patients were monitored using: electrocardiogram, non-invasive arterial pressure, pulse oximeter and bispectral index (BIS). Induction of general anesthesia was performed by injecting glycopyrrolate $(0.2 \mathrm{mg})$ and $2 \%$ lidocaine $(30 \mathrm{mg})$. Propofol, $2 \%$ (Fresofol $^{\circledR}$, Fresenius Kabi, Korea Ltd, Korea) and remifentanil (Ultiva ${ }^{\circledR}$, GlaxoSmithKlein, UK), which was diluted to $20 \mu \mathrm{g} / \mathrm{ml}$ with a Target Controlled Infusion device (Orchestra ${ }^{\circledR}$, Fresenius vial, France) until the effective concentrations became $4.0 \mu \mathrm{g} / \mathrm{ml}$ and $4.0 \mathrm{ng} / \mathrm{ml}$, respectively. After unconsciousness was confirmed, an intravenous injection of $0.15 \mathrm{mg} / \mathrm{kg}$ of vecuronium was administered and then endotracheal intubation was performed using an endotracheal tube with an inner diameter (ID) size of $7.0 \mathrm{~mm}$ after confirming that the muscle was sufficiently relaxed by a nerve stimulator (TOF watch ${ }^{\circledR}$, Organon, Ireland). Anesthesia was maintained with $50 \%$ oxygen in air with a total fresh gas flow of $3 \mathrm{~L} / \mathrm{min}$ controlled by mechanical ventilation. The $2 \%$ propofol and remifentanil concentrations were regulated to maintain a BIS of 40 to 60 and blood pressure was kept to within $20 \%$ of the blood pressure measured when arriving in the operation room. Also, the end-tidal carbon dioxide partial pressure was maintained within the range of 30 to $40 \mathrm{mmHg}$.

When the vital signs were stabilized after induction of anesthesia, the US-TAP block was performed under ultrasound (SonoSite $180^{\circledR}$, SonoSite, USA) guidance. The block technique was similar to the method described by Hebbard and colleages [17] and all US-TAP blocks were performed by one investigator. After sterilizing the abdominal part between the xiphoid process and pubic symphysis with povidone-iodine, a high frequency linear probe (L38, 10-5 MHz, SonoSite, USA) coated 
with sterilized gel was placed transversely on the mid-axillary line between the iliac crest and the subcostal margin of the $12^{\text {th }}$ rib on the right side. Views were considered satisfactory, if subcutaneous tissue, external oblique abdominal muscle, internal oblique abdominal muscle, transversus abdominis muscle, the transversus abdominis plane and the peritoneal cavity were identified. Then a 20 gauge, $100 \mathrm{~mm}$ needle (Stimuplex ${ }^{\circledR}$ A, B. Broun Melsungen AG, Germany) was introduced through the skin at the medial end of the ultrasound probe and inserted in-plane under real-time ultrasound guidance to lie between the internal oblique and the transversus abdominis muscles with the tip in the mid-axillary line (Fig. 1A). The correct position of the needle in the transversus abdominis fascial plane (between the internal oblique and the transversus abdominis muscle) was established using direct ultrasound inplane visualization and distention of the fascial plane by a $1 \mathrm{ml}$ test injection of $0.375 \%$ ropivacaine (Fig. 1B). After checking the diffusion of local anesthetics between the fascial planes, injection of the remaining $19 \mathrm{ml}$ was given. A similar procedure was performed on the contralateral side of the abdomen with the injection of another $20 \mathrm{ml}$ of the $0.375 \%$ ropivacaine.

An investigator, blinded to group allocation, assessed the presence and the severity of pain, drowsiness, nausea and itch. Data were collected in the PACU (0) and at 2, 6, 10, 24 and 48 hours after admission to the PACU. All patients were asked to give scores for their pain at rest and on movement (knee flexion) as well as the degree of nausea and itch at each time point. Also, the degree of drowsiness was assessed by the investigator at the same time points. Pain severity was measured using a verbal numerical rating scale (VNRS: $0=$ no pain, $10=$ very severe pain) pain score. Drowsiness was assessed by the investigator using a categorical scoring system (awake and alert $=0$; quietly awake $=1$; asleep but easily roused $=2$; deep sleep $=3$ ). Also, Nausea and itch were measured using a categorical scoring system $($ none $=0$; mild $=1$; moderate $=2$; severe $=3$ ).

Patients postoperative pain was controlled by an IV-
PCA consisting of ketorolac (90 mg), sufentanil (200 $\mu \mathrm{g})$, ramosetron hydrochloride $\left(0.3 \mathrm{mg}\right.$; Nasea ${ }^{\circledR}$, Astellas, Korea) and $0.9 \%$ normal saline in a total volume of $120 \mathrm{ml}$. The IV-PCA maintenance dose, bolus dose, and lockout time were $1.5 \mathrm{ml} / \mathrm{h}$, $1.5 \mathrm{ml}$, and 15 minutes respectively, according to standard regimen of out unit.

If the VNRS pain score was higher than 6 in the PACU, fentanyl $(25 \mu \mathrm{g})$ was injected and if it was lower than 6 , or if the patient wanted an analgesics, ketorolac ( $30 \mathrm{mg}$ ) was injected. When the patient complained of pain associated with shivering, pethidine (25 mg) was injected in the PACU. Also, when the VNRS pain score was 6 or the patient wanted a pain killer in the ward, pethidine $(25 \mathrm{mg}$ ) or ketorolac ( $30 \mathrm{mg}$ ) were injected as this is a standard postoperative analgesic regimen of the Department of Obstetrics and Gynecology of our institution.

We assessed the total injected dose of fentanyl, ketorolac and pethidine in the PACU and ward as well as compared the total infused volume of IV-PCA during the first postoperative 48 hour.

The primary outcome measures in this study were the VNRS pain score at rest in the PACU. The secondary outcome measures included the VNRS pain score on movement in the PACU and at 2, 6, 10, 24, 48 hour after admission on the PACU (both at rest and on movement) and total supplement analgesic consumption in the first 48 hours after surgery. The patient's satisfaction with the pain management was measured using a VNRS ( $0=$ very unsatisfied, $10=$ completely satisfied) pain score.

The sample size was calculated based on data from an initial pilot study, in which we estimated the average VNRS pain score at rest in the PACU after surgery to be $8.10 \pm 1.45$ and we assumed that reduction of a VNRS pain score of 3 or more would be clinically significant. We calculated that 13 patients would be required per group for an experimental design with an $\alpha=0.05$ and $\beta=0.2$, and elected 16 patients per group considering the patients that might be excluded.

Statistical analyses were performed using the statistical
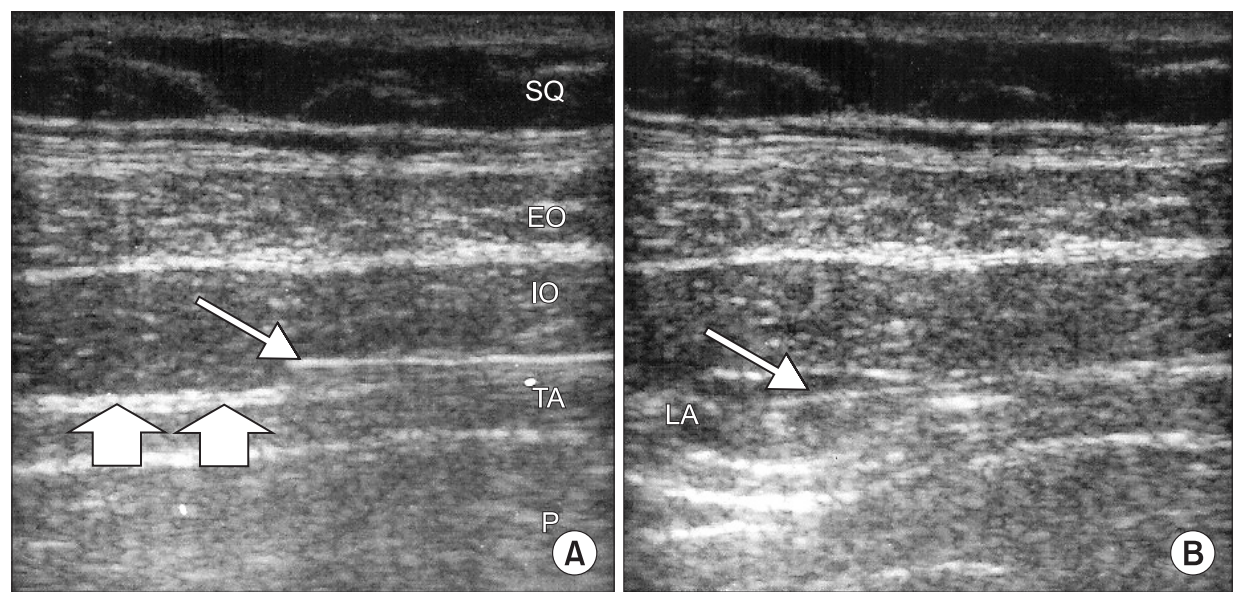

Fig. 1. Sonographic anatomy of the USguided TAP block. Images show the lateral abdominal wall using a probe held in the mid-axillary line in the axial plane. The right of image is anterior. (A) Narrow arrow: needle, SQ: subcutaneous tissue, EO: external oblique muscle, IO: internal oblique muscle, TA: transversus abdominis muscle, P: peritoneal cavity. Broad arrow, transversus abdominis plane. (B) Narrow arrow: needle, LA: local anesthetic. 
program, SPSS, v 12.0. The continuous numerical data were tested for normality using the Shapiro-Wilk test. Normally distributed data were analyzed by a Student's t-test and nonnormally distributed measurements were analyzed by the Mann-Whitney U-test. Therefore, the patient' height and BMI were compared between the two groups using the Student's t-test. Patient's age, weight, and the operation time were compared by the Mann-Whitney U-test., The ASA physical status, history of previous abdominal surgery, the surgeon and incision methods were compared appropriately between the two groups by chi-square analysis or Fisher's exact test. The VNRS pain scores, the total dose of ketorolac, fentanyl, and pethidine used in the PACU, the total amount of ketorolac, pethidine used in the ward and the total infused dose of IV-PCA during the first 48 hours were compared using a Mann-Whitey U-test. Categorical variables were statistically analyzed by chisquare analysis or Fisher's exact test. Data were considered statistically significant when $\mathrm{P}<0.05$.

Table 1. Patient Characteristics

\begin{tabular}{lcc}
\hline \multicolumn{1}{c}{ Group } & $\begin{array}{c}\text { Control } \\
(\mathrm{n}=16)\end{array}$ & $\begin{array}{c}\text { US-TAP block } \\
(\mathrm{n}=16)\end{array}$ \\
\hline Age (yr) & $43.4 \pm 12.9$ & $41.3 \pm 9.3$ \\
Weight (kg) & $57.2 \pm 10.4$ & $62.7 \pm 10.1$ \\
Height (cm) & $154.0 \pm 3.3$ & $157.1 \pm 5.7$ \\
BMI (kg/m $\left.{ }^{2}\right)$ & $24.1 \pm 4.2$ & $25.3 \pm 3.3$ \\
ASA physical status (I/II) & $10 / 6$ & $12 / 4$ \\
Previous abdominal surgery & 8 & 7 \\
Surgeon (K, J, P) & $6 / 9 / 1$ & $1 / 11 / 4$ \\
Incision (Cherney/Pfannenstiel) & $9 / 7$ & $12 / 4$ \\
Operation time (min) & $116.9 \pm 68.3$ & $112.8 \pm 73.8$ \\
\hline
\end{tabular}

Results are expressed as means \pm sd or numbers of patients. There were no significant differences between groups. US-TAP: ultrasoundguided transversus abdominis plane, BMI: body mass index, ASA: American Society of Anesthesiologists.

Table 2. Postoperative Analgesic Requirements

\begin{tabular}{|c|c|c|}
\hline Group & $\begin{array}{l}\text { Control } \\
(n=16)\end{array}$ & $\begin{array}{l}\text { US-TAP block } \\
\qquad(\mathrm{n}=16)\end{array}$ \\
\hline \multicolumn{3}{|l|}{ Analgesics in PACU } \\
\hline Ketorolac (mg) & $18.8 \pm 15.0$ & $15.0 \pm 15.5$ \\
\hline Pethidine (mg) & $3.1 \pm 8.5$ & $6.3 \pm 11.2$ \\
\hline Pethidine $^{\dagger}$ (mg) & $0.4 \pm 1.1$ & $0.8 \pm 1.5$ \\
\hline Fentanyl ( $\mu \mathrm{g})$ & $62.5 \pm 35.4$ & $20.3 \pm 20.9 *$ \\
\hline Fentanyl $^{\dagger}$ (mg) & $6.3 \pm 3.5$ & $2.0 \pm 2.1 *$ \\
\hline \multicolumn{3}{|l|}{ Analgesics in ward } \\
\hline Ketorolac (mg) & $3.75 \pm 10.3$ & $5.6 \pm 12.1$ \\
\hline Pethidine (mg) & $56.3 \pm 34.8$ & $21.9 \pm 28.7 *$ \\
\hline Pethidin $^{\dagger}$ (mg) & $7.5 \pm 4.6$ & $2.9 \pm 3.8^{*}$ \\
\hline Total infused PCA volume (ml) & $107.5 \pm 31.1$ & $101.7 \pm 24.8$ \\
\hline
\end{tabular}

Results are expressed as means \pm sd. US-TAP: ultrasound-guided transversus abdominis plane, PACU: postanesthesia care unit, PCA: patient-controlled analgesia. ${ }^{*} \mathrm{P}$ value $<0.05$ compared as Group Control. ${ }^{\dagger}$ Value converted to a morphine equivalent dose.

\section{Results}

With 16 patients per group, a total of 32 patients were entered into this study. The groups were comparable in terms of age, weight, height, BMI, ASA physical status, history of previous abdominal surgery, surgeon, incision methods and operation time; and there were no statistically significant differences between the two groups (Table 1). There were no significant differences in the incidence of drowsiness, nausea, and itch between groups at any time point.

The US-TAP block significantly reduced the total consumption of fentanyl, which was injected in the PACU and the amounts of pethidine, which was offered in the ward. When comparing the amounts of fentanyl and pethidine after converting to a morphine equivalent dose, there were also significant differences between the two groups. Nevertheless, there was no significant difference in the total infused dose of IV-PCA during the first 48 postoperative hours (Table 2).

Table 3. Pain Intensity at Different Postoperative Period

\begin{tabular}{lll}
\hline \multicolumn{1}{c}{ Group } & $\begin{array}{c}\text { Control } \\
(\mathrm{n}=16)\end{array}$ & $\begin{array}{c}\text { US-TAP block } \\
(\mathrm{n}=16)\end{array}$ \\
\hline $\begin{array}{l}\text { VNRS at rest } \\
\text { PACU }\end{array}$ & \\
$2 \mathrm{hrs}$ & $8.4 \pm 1.3$ & $5.2 \pm 3.1^{*}$ \\
$6 \mathrm{hrs}$ & $5.2 \pm 2.4$ & $3.0 \pm 2.4^{*}$ \\
$10 \mathrm{hrs}$ & $4.1 \pm 2.4$ & $2.6 \pm 2.5$ \\
$24 \mathrm{hrs}$ & $3.6 \pm 2.3$ & $2.3 \pm 2.4$ \\
$48 \mathrm{hrs}$ & $2.2 \pm 1.9$ & $0.9 \pm 1.5^{*}$ \\
VNRS with movement & $1.6 \pm 1.2$ & $0.4 \pm 0.7$ \\
PACU & & \\
$2 \mathrm{hrs}$ & $8.6 \pm 1.0$ & $5.5 \pm 3.1^{*}$ \\
$6 \mathrm{hrs}$ & $5.7 \pm 2.4$ & $3.4 \pm 2.5^{*}$ \\
$10 \mathrm{hrs}$ & $4.9 \pm 2.5$ & $3.3 \pm 2.5$ \\
$24 \mathrm{hrs}$ & $4.3 \pm 2.3$ & $2.8 \pm 2.5$ \\
$48 \mathrm{hrs}$ & $3.1 \pm 2.0$ & $1.4 \pm 1.6^{*}$ \\
\hline
\end{tabular}

Results are expressed as means \pm sd. US-TAP: ultrasound-guided transversus abdominis plane, VNRS: verbal numerical rating scale, PACU: postanesthesia care unit. *P value $<0.05$ compared as Control Group.

Table 4. Patients' Satisfaction Measured using VNRS

\begin{tabular}{lll}
\hline Group & $\begin{array}{c}\text { Control } \\
(\mathrm{n}=16)\end{array}$ & $\begin{array}{c}\text { US-TAP block } \\
(\mathrm{n}=16)\end{array}$ \\
\hline PACU & $2.3 \pm 2.5$ & $5.2 \pm 3.5^{*}$ \\
$2 \mathrm{hrs}$ & $4.6 \pm 2.5$ & $6.8 \pm 2.7^{*}$ \\
$6 \mathrm{hrs}$ & $5.8 \pm 2.4$ & $7.3 \pm 2.7$ \\
$10 \mathrm{hrs}$ & $6.6 \pm 2.3$ & $8.0 \pm 2.3^{*}$ \\
$24 \mathrm{hrs}$ & $6.9 \pm 2.9$ & $8.9 \pm 2.5^{*}$ \\
$48 \mathrm{hrs}$ & $7.9 \pm 2.5$ & $9.2 \pm 1.8^{*}$ \\
\hline
\end{tabular}

Results are expressed as mean \pm sd. PACU: postanesthesia care unit, US-TAP: ultrasound-guided transversus abdominis plane. *P value $<$ 0.05 compared as Control Group. 
The VNRS pain score of the US-TAP Block group was significantly lower in the PACU and at 2, 24 hour postoperatively at rest in addition to being lower in the PACU and at 2, 24, 48 postoperative hours on movement (Table 3 ). Also, patient's satisfaction was significantly higher in the PACU and at 2, 10, 24,48 hours after the operation in the US-TAP Block group with respect to pain control (Table 4 ).

\section{Discussion}

This study demonstrates that a US-TAP block provides effective analgesia, when used as part of a multimodal analgesic regimen in patients undergoing gynecological surgery via a transverse lower abdominal skin incision. There was no significant difference in the total infused dose of IV-PCA during the first 48 postoperative hours. But, the US-TAP block reduced postoperative fentanyl and pethidine consumptions and this result means that the US-TAP block can confer a benefit in the management of postoperative pain in addition to decreasing the incidence of side effects such as nausea, vomiting, dizziness, and itch by reducing the requirements of opioid. In addition, the VNRS pain scores assessed in the US-TAP Block group were significantly lower than the Control group in the PACU and at 2, 24 hours after the operation at rest as well as in the PACU and at 2, 10, 24 and 48 postoperative hours on movement. This demonstrates represents that the US-TAP block provides effective analgesia in the initial postoperative stage. Kang et al. [1] carried out a survey about postoperative pain and they reported that nearly $70 \%$ of patients expected more than a moderate degree of pain and most patients who were asked to answer the questionnaires had pain mainly in the early postoperative period. So, they suggested that pain control should be focused more on the initial postoperative stage than at later periods.

We believe there are two reasons why there was no difference in the infused volume via IV-PCA between two groups. First, it is supposed that the patients who had an US-TAP block pressed the bolus button despite mild postoperative pain. In other words, we cannot assure that the patients used the bolus button under the same degree of pain because we did not assess how much pain made them to press the bolus button. This suggests that all patients in each group might press the bolus button with similar frequency to reduce the early postoperative pain although the VNRS pain scores are different between the two groups. Second, the IV-PCA regimen dose might not be enough to control of postoperative pain.

The US-TAP block has advantages of being performing accurately and safely in a short time because the procedure is implemented by watching real time images of the ultrasound monitor and the target site has no vital anatomical structures like large vessels and the spinal cord. Moreover, somatic pain can be fully managed by using a US-TAP block but not the epidural analgesic method as somatic pain comes from a narrow dermatome in the surgery with transverse lower abdominal skin incision.

The adverse effects pertaining to the TAP block have been reported in the literature. Farooq and Carey [18] reported a case of liver trauma with a blunt regional anesthesia needle while performing a TAP block. But they carried out the block solely using the blind "double-pop" technique, so they could not evaluate the anatomical structures and the exact location of the needle tip. There is a case of US-TAP block causing liver trauma and peritonitis in a patient undergoing an inguinal hernia repair [19]. This is the first reported case of a US-TAP block causing a significant postoperative complication even when ultrasound was used. But the damage occurred due to a failure to accurately imaging the entire needle during the right-sided needle placement, resulting in excessive depth of penetration. Therefore, if we check the exact direction and depth of the needle watching real time images of the ultrasound monitor, this problem can be prevented sufficiently. In our study, there were no complications during the procedure or after the block.

The optimal effective volumes and concentrations of injected local anesthetics are not established and not much is known about the analgesic duration and the accurate onset time. Therefore, it is necessary to study these parameters during a TAP block. If research on the degree of pain killing via continuous infusion of analgesics is done on the TAP block through a catheter placed into transversus abdominis plane like epidural analgesia, it may support the usefulness of TAP block. The point of time to implement the block also needs to be investigated. In this study, we performed the US-TAP block after induction of general anesthesia before surgical incision. The reason for reducing the early VNRS pain scores could be interpreted as a kind of preemptive analgesic effect. Based on our experience, there are no reliable data or studies but it seems that the effect of the US-TAP block is more marked on reducing the degree of pain when the block is carried out in patients who are alert and conscious in recovery room rather than after induction. Therefore, high quality care can be offered to patients if research is performed studying the reduction of pain according to the timing of block implementation.

Attention should be given to the opinion of Tornero-Campello, where the TAP block should be compared with epidural analgesia for postoperative pain control after abdominal surgery [20]. He suggested that "epidural analgesia must be considered 'standard care' for patients receiving abdominal surgery". But we think that it is more reasonable to apply the epidural block on the basis of the data including the patient's condition, type of surgical procedure, and risk-benefits that 
may be occur after a neuraxial blockade rather than to provide it uniformly to all patients undergoing abdominal surgery. Also, it is the responsibility of the anesthesiologist to decide the most appropriate method for controlling the postoperative pain in their patients. There are a few surgical procedures using the US-TAP block technique so far and the US-TAP block could be applied widely. If this block is developed through more research, it may be increasingly used in the field for treating patient's pain.

In conclusion, the US-TAP block has opioid-sparing effects, reduces postoperative pain and improves the satisfaction of pain relief in patients when used a part of a multimodal analgesic regimen. Further the procedure is relatively easy and simple without serious complications. Therefore, if the USTAP is used in patients undergoing gynecological surgery via a transverse lower abdominal skin incision appropriately, it can be a useful pain control method with a multimodal analgesic regimen

\section{References}

1. Kang H, Hong SH, Kim H, Kim ES, Shin HJ. Pre- and post-operative patient's reactions to postoperative pain. Chungbuk Med J 2007; 17: 182-9.

2. Kehlet H. Surgical stress: the role of pain and analgesia. Br J Anaesth 1989; 63: 189-95.

3. Weissman C. The metabolic response to stress: an overview and update. Anesthesiology 1990; 73: 308-27.

4. Woodhouse A, Mather LE. The effect of duration of dose delivery with patient-controlled analgesia on the incidence of nausea and vomiting after hysterectomy. Br J Clin Pharmacol 1998; 45: 57-62.

5. Cameron CM, Scott DA, McDonald WM, Davies MJ. A review of neuraxial epidural morbidity: experience of more than 8,000 cases at a single teaching hospital. Anesthesiology 2007; 106: 997-1002.

6. Cook TM, Counsell D, Wildsmith JA; Royal College of Anaesthetists Third National Audit Project. Major complications of central neuraxial block: report on the Third National Audit Project of the Royal College of Anaesthetists. Br J Anaesth 2009; 102: 179-90.

7. Chung MY, Kim CJ. The effect of femoral nerve block combined with intravenous patient-controlled analgesia after a unilateral total knee replacement. Korean J Anesthesiol 2008; 55: 591-601.
8. Kim CS, Lim KJ, Chung CD, Lee EY. The analgesic effect of continuous suprascapular nerve block after arthroscopic shoulder surgery. Korean J Anesthesiol 2004; 47: 92-5.

9. Abrahams MS, Aziz MF, Fu RF, Horn JL. Ultrasound guidance compared with electrical neurostimulation for peripheral nerve block: a systematic review and meta-analysis of randomized controlled trials. Br J Anaesth 2009; 102: 408-17.

10. Niraj G, Searle A, Mathews M, Misra V, Baban M, Kiani S, et al. Analgesic efficacy of ultrasound-guided transversus abdominis plane block in patients undergoing open appendicectomy. Br J Anaesth 2009; 103: 601-5.

11. Ra YS, Kim CH, Lee GY, Han JI. The analgesic effect of the ultrasoundguided transverse abdominis plane block after laparoscopic cholecystectomy. Korean J Anesthesiol 2010; 58: 362-8.

12. Carney J, McDonnell JG, Ochana A, Bhinder R, Laffey JG. The transversus abdominis plane block provides effective postoperative analgesia in patients undergoing total abdominal hysterectomy. Anesth Analg 2008; 107: 2056-60.

13. McDonnell JG, O'Donnell B, Curley G, Heffernan A, Power C, Laffey JG. The analgesic efficacy of transversus abdominis plane block after abdominal surgery: a prospective randomized controlled trial. Anesth Analg 2007; 104: 193-7.

14. McDonnell JG, Curley G, Carney J, Benton A, Costello J, Maharaj CH, et al. The analgesic efficacy of transversus abdominis plane block after cesarean delivery: a randomized controlled trial. Anesth Analg 2008; 106: 186-91.

15. Belavy D, Cowlishaw PJ, Howes M, Phillips F. Ultrasound-guided transversus abdominis plane block for analgesia after Caesarean delivery. Br J Anaesth 2009; 103: 726-30.

16. Rafi AN. Abdominal field block: a new approach via the lumbar triangle. Anaesthesia 2001; 56: 1024-6.

17. Hebbard P, Fujiwara Y, Shibata Y, Royse C. Ultrasound-guided transversus abdominis plane (TAP) block. Anaesth Intensive Care 2007; 35: 616-7.

18. Farooq M, Carey M. A case of liver trauma with a blunt regional anesthesia needle while performing transversus abdominis plane block. Reg Anesth Pain Med 2008; 33: 274-5.

19. Lancaster $P$, Chadwick M. Liver trauma secondary to ultrasoundguided transversus abdominis plane block. Br J Anaesth 2010; 104: 509-10.

20. Tornero-Campello G. Transversus abdominis plane block should be compared with epidural for postoperativ analgesia after abdominal surgery. Anesth Analg 2007; 105: 281-2. 